\section{PWE-077 BILE ACID MALABSORPTION A DIFFERENTIAL DIAGNOSIS FOR PATIENTS PRESENTING WITH DIARRHOEA PREDOMINANT IRRITABLE BOWEL SYNDROME TYPE SYMPTOMS?}

doi:10.1136/gut.2011.239301.340

M Kurien, ${ }^{1,}$ K E Evans, ${ }^{1}$ J S Leeds, ${ }^{1}$ A D Hopper, ${ }^{1}$ A Harris, ${ }^{2}$ M Hanney, ${ }^{2}$ D S Sanders' ${ }^{1}$ Department of Gastroenterology, Royal Hallamshire Hospital, Sheffield Teaching Hospitals, Sheffield, UK; ${ }^{2}$ Department of Medical Physics, Royal Hallamshire Hospital, Sheffield Teaching Hospitals, Sheffield, UK

Introduction Irritable Bowel Syndrome (IBS) should be a positive diagnosis rather than a diagnosis of exclusion. However, in unselected patients presenting with IBS type symptoms it is important to recognise other differential diagnosis. Bile acid malabsorption (BAM) has been reported as a possible cause of diarrhoea predominant (D)-IBS type symptoms. For this reason we aimed to determine how commonly patients with D-IBS type symptoms had a diagnosis of BAM as demonstrated by a positive SeHCAT ${ }^{75}$ Selenium HomotauroCholic Acid Taurine) test (retention $<10 \%$ at 7 days).

Methods (Group A) A retrospective analysis was undertaken of patient's records for all patients who underwent a SeHCAT scan in a 9-year period between 2001 and 2010 in a tertiary Gastroenterology Department. Patient demographics, symptoms, previous medical and surgical history, as well as laboratory investigations (including full blood count, serum folate, vitamin $B_{12}$, ferritin and albumin) were analysed. Statistical analysis was performed using SPSS (version 19.0 for windows) with $\chi^{2}$ analysis used to compare the data. Concurrently a cohort of patients (Group B) with D-IBS type symptoms (fulfilling Rome II criteria) was examined to determine the potential utility of SeHCAT test.

Results In Group A of the 415 patients who underwent a SeHCAT, 368 patient records were located and analysed (mean age 49.3, 114 male). 153 patients (41.6\%) had a positive result. The mean time from first hospital visit to SeHCAT result was 32 weeks. Patient characteristics predictive of a positive SeHCAT were: terminal ileal Crohn's $(p<0.01)$, terminal ileal resection $(p<0.01)$, inflammatory bowel disease $(p<0.01)$, previous small bowel surgery $(\mathrm{p}<0.01)$ and previous cholecystectomy $(p<0.01) .47$ of the 153 patients $(30.7 \%)$ of the patients 
referred with D-IBS type symptoms had a positive SeHCAT scan. In Group B the D-IBS control cohort $(n=316$, mean age 46.1 years, 96 males) only $6 / 316(1.9 \%)$ patients had been referred for a SeHCAT scan ( $<<0.001$ compared to Group A) meaning that potentially 96 patients could have been missed.

Conclusion BAM is common and should be considered earlier in patients with predisposing factors (including inflammatory bowel disease, terminal ileal disease, previous small bowel surgery and post cholecystectomy). Consideration should also be given for BAM when investigating unselected patients with D-IBS type symptoms.

Competing interests None.

Keywords Bile Acid Malabsorption, Irritable Bowel Syndrome, SeHCAT. 